\title{
Management of Occult Breast Carcinoma: A Meta-Analysis Study
}

\author{
OSAMA A. AL-ATRASH, M.D.; KARIM FAHMY, M.D. and AHMED S. EL-SAYED ABD EL-RAOUF, M.Sc. \\ The Department of General Surgery, Faculty of Medicine, Ain Shams University
}

\begin{abstract}
Background: Breast cancer is the commonest primary neoplasm affecting females in Egypt, it is considered the third leading cause of cancer related deaths in Egypt, it has been established during past decades, that surgery, radiotherapy and chemotherapy are corner stones in treatment of breast cancer.
\end{abstract}

Aim of Study: Is to locate relevant studies comparing different lines of management most commonly used in treatment of occult breast cancer that are published till now, revising all these studies, and designing a meta-analysis study to assess the overall result and to highlight the different lines of management of occult breast cancer and to assess recurrence rate and prognosis.

Material and Methods: In this meta-analysis, we systematically investigated the present therapeutic options in patients diagnosed with $\mathrm{OBC}$, targeting a definition of the surgical approach associated with best surgical and recurrence outcomes. 10 studies were eligible for inclusion in our metaanalysis, all were published in the period from inception till 2020 , revising all these data, none the less designing metaanalysis study to evaluate the overall result and to highpoint the different surgical approaches in management of occult breast cancer and to evaluate recurrence rate and prognosis.

Results: A total of 837 patients were included in metaanalysis, with median age 54 years range from 44 to 59.3 years old with median follow-up period 67.7 months, compared to other meta-analysis conducted by Macedo et al., had seven studies included with total number of 241 patients eligible for analysis, median age were 55 years ranged between 5059 years old.

Conclusion: There was no statistically difference between BCS + ALND and Mastectomy + ALND surgical approaches in terms of recurrence including loco regional and distant also there was difference in survival (OS and DFS) between BCS + ALND and Mastectomy + ALND surgical approaches.

Key Words: Occult Breast Carcinoma-Breast Cancer.

Correspondence to: Dr. Ahmed S. El-Sayed Abd El-Raouf, E-Mail: ahmedsamy2014.as33@gmail.com

\section{Introduction}

CARCINOMA of the breast is the commonest cancer in female and the second most cause of death in cancer related deaths. It is estimated that there will be 246,660 new cases of female breast cancer and an estimated 40,450 patient will die of this disease in the United States in 2016 [1].

There are several independent but interrelated prognostic factors predictive of recurrence and survival in breast cancer. These include axillary nodal status, histopathology, steroid receptors, proliferative rate, DNA ploidy, and oncogene amplification. S-phase fraction can also be used to help define the high-risk patient. Axillary nodal status has been the traditional mainstay predictor for recurrence and survival in primary breast cancer. In addition, the presence of the estrogen and progesterone receptors has correlated with longer disease-free interval and overall survival in stage I and II breast cancer. Finally, tumors that amplify or over express the HER-2 gene may have a higher risk of relapse [2].

Screening and improved adjuvant therapy have led to reduced breast cancer mortality in the United States, highlighting the importance of appropriate detection and management of the disease. The U.S. Preventive Services Task Force recommends screening using mammography every two years in women between the ages of 50 and 74 [3].

Breast cancer is comprised of a number of complex and heterogeneous subtypes with differing clinical behavior and outcomes. Most clinical decisions are currently based on tumor expression of the Estrogen Receptor (ER), Progesterone Receptor (PR), and human epidermal growth factor receptor 2 (HER 2). These biomarkers have prognostic and predictive significance in breast cancer 
and have important implications for tumor growth and metastatic patterns [4].

Distant spread of breast cancer results in poor survival outcome and the site of the distance recurrence are also important to predict the clinical outcome [5].

It has been noted that there is a significant difference in survival among the molecular subtypes of breast cancer [6]. However data are limited concerning differences in distant recurrence sites between the breast cancer subtypes [7].

A few studies have described different distant metastatic pattern according to molecular subtypes. The risk associated with a positive family history of breast cancer is strongly affected by the number of female first-degree relatives with and without cancer. As an example, in a pooled analysis using data from over 50,000 women with breast cancer and 100,000 controls, the risk of breast cancer was increased almost two folds if a woman had one affected first degree relative, increased three folds if she had two affected first degree relatives [8].

In addition to a family history of breast cancer, the age at diagnosis of the affected first degree relative also influences the risk for breast cancer. Women have a threefold higher risk if the first degree relative was diagnosed before age 30 , but only 1.5 -fold increased if the affected relative was diagnosed after age 60 [9]

Routine pathologic evaluation remains the most critical element in determining the prognosis of patients with breast cancer. Among the potent prognostic factors available are lymph node status, tumor size and histologic grade, histologic tumor type, and lymphatic vascular invasion [10].

\section{Occult Breast Cancer:}

Occult Breast Cancer (OBC) was first described by Halsted et al., who reports three cases presenting with enlarged axillary lymph nodes and no breast mass was detectable until 1-2 years later [11].

Traditionally, OBC is characterized by metastasis to the axillary lymph nodes with no detectable breast mass at the initial presentation [12]. All systematic reviews and meta-analyses of the diagnoses and therapy of OBC to date are based on this definition. The morbidity of OBC is approximately $0.3 \%$ to $0.8 \%$ globally, and about $0.7 \%$ in China [13]
Obviously, with the development of imaging techniques, the morbidity of OBC will continue to gradually decrease. Ultrasonography and mammography are very popular modalities for imaging of breast cancer. Besides Magnetic Resonance Imaging (MRI), positron emission tomography computed tomography of the breast is also often applied in clinical practice worldwide. As a result, some OBC cases will be revised once a palpable breast mass is detected by these advanced imaging examinations [14].

Notably, most enlarged axillary lymph nodes detected by finger examination and/or mammography are in the preliminary stage. However, the choice of treatment may change once an enlarged axillary lymph node begins to show signs of malignancy [15].

The most frequent cause of metastasis of breast cancer to the axillary lymph nodes is unique to the origin of the breast cancer. Besides, other primary foci, including axillary lymphoma, malignant blood disease, lung cancer, ovarian carcinoma, head and neck squamous cell carcinoma, colon carcinoma, gastric cancer and thyroid cancer etc. can also result in enlargement of the axillary lymph nodes [16].

Therefore, based on past-experience, detailed family history and intensive physical examination are becoming more and more crucial to identify the essential cause of enlarged axillary lymph nodes. Patients with OBC usually have no detectable breast mass, but rather enlarged axillary lymph nodes. For detection of metastatic lymph nodes derived from breast cancer, mammography and ultrasonography of the breast and bilateral axillary lymph nodes, supraclavicular lymph nodes, and internal mammary lymph nodes are highly recommended [17].

If these examinations of the breast are still not sufficient to identify the position of the carcinoma, biopsy of enlarged axillary lymph nodes by fineneedle aspiration or blunt needle aspiration will be necessary [15].

Approximately 1.6 million women in the US undergo breast biopsies annually and nearly onequarter of the biopsies demonstrate the origin of invasive breast cancer. It is very important that fine-needle biopsy is performed by an experienced pathologist and blunt-needle biopsy requires an adequate tissue sample to accurately determine the histological features of the foci. If a definitive 
diagnosis cannot be determined by the tissue biopsy, the lymph nodes should be enucleated [18]

Once of breast cancer to the lymph nodes is confirmed, conservative examination by mammography is required. MRI of the bilateral breasts is an alternative choice when there are no specific findings by mammography. MRI is extremely useful to locate the primary focus in suspected OBC cases with negative results by mammography and/or B-type ultrasonography. The sensitivity ( $85 \%$ to $100 \%)$ and specificity $(37 \%$ to $77 \%)$ of MRI are greater than those of traditional finger examination and other imaging techniques [18].

Olson et al., compared the efficacy of MRI to that of traditional imaging methods for diagnosis of OBC in 40 patients and found that MRI accurately located the primary foci in resected specimens after modified radical oncology breast surgery in $21(95 \%)$ of 22 suspected cases. Of 12 cases with negative MRI results, five underwent breast surgery, but no primary lesion was detected in four $(80 \%)$ of the cases [19].

These findings substantiate the high sensitivity of MRI and its value in clinical application. BreastSpecific y-Imaging (BSGI) is another physiological imaging tool used to detect breast cancer, which uses a radiotracer, $99 \mathrm{mT}$ csestamibi, to identify physiologic differences between malignant and normal breast tissue [19].

BSGI detects occult breast cancer in women at increased risk at a rate of 16.5 cancer detected per 1,000 women screened, higher than the rate reported for both MRI and screening ultrasound. Furthermore, BSGI can also enhance the ability to detect breast cancer in women at increased risk compared with mammography alone. Eleven of 14 (78.6\%) mammographically occult cancers were detected in patients with heterogeneous or extremely dense breast tissue (BI-RADS c or d). Based on this evidence, it is necessary to upgrade conventional examinations for OBC to tissue punctures or MRIguided direct needle biopsy to improve diagnostic reliability [20].

Kuhl et al., reported that MRI combined with finger examination, B-type ultrasonography, and $\mathrm{X}$-ray imaging was useful for diagnosis of OBC, successfully increasing the detection rate up to $98 \%$, with a sensitivity of up to $96 \%$ and a specificity of up to $100 \%$. Moreover, the positive prophylactic value increased to $100 \%$. While the negative prophylactic value while increased to $97 \%$ [20]

\section{Aim of the study:}

The aim is to locate relevant studies comparing different lines of management most commonly used in treatment of occult breast cancer that are published till now, revising all these studies, and designing a meta-analysis study to assess the overall result and to highlight the different lines of management of occult breast cancer and to assess recurrence rate and prognosis.

\section{Material and Methods}

We performed this systematic review and metaanalysis in accordance to the recommendations of the Preferred Reporting Items for Systematic Reviews and Meta-Analyses (PRISMA) statement and Meta-analysis of Observational Studies in Epidemiology (MOOSE) statement. PRISMA and MOOSE are reporting checklists for Authors, Editors, and Reviewers of Meta-analyses of interventional and observational studies. According to International committee of medical journal association (ICJME), reviewers must report their findings according to each of the items listed in those checklists [21]

\section{Study selection and eligibility criteria:}

The present review included studies that fulfilled the following criteria: (1) Studies that included adults' patients with Occult Breast Cancer (OBC). OBC is defined as adenocarcinoma that presented with axillary metastases in the absence of primary breast tumor on physical examination, imaging, or pre-operative biopsy, (2) Studies that assessed the safety and effectiveness of different management lines for OBC, (3) Studies that reported any of the following outcomes: Clinical response, survival, recurrence, metastasis and complication, (4) Studies that were Randomized Controlled Trials (RCTs), comparative studies, or prospective cohort studies.

We excluded review articles, non-English studies, theses, dissertations and conference abstracts, and trials with unreliable date for extraction.

\section{Search strategy and screening:}

An electronic search was conducted from the inception till June 2020 in the following bibliographic databases: Medline via PubMed, SCOPUS, Cochrane Central Register of Controlled Trials (CENTRAL), and Web of Science to identify relevant articles. We used different combinations of the following queries: "Occult breast cancer"; "occult primary breast cancer"; "occult breast neoplasm"; "mastectomy"; and keywords "primary 
axillary metastases"; "axillary adenopathy"; "axillary lymph node dissection" with limits "human".

\section{Screening:}

Retrieved citations were imported into EndNote X7 for duplicates removal. Subsequently, unique citations were imported into an Excel sheet and screened by two independent reviewers; the screening was conducted in two steps: Title and abstract screening, followed by a full-texts screening of potentially eligible records.

\section{Data extraction:}

Data entry and processing were carried out using a standardized Excel sheet and reviewers extracted the data from the included studies. The extracted data included the following domains: (1) Summary characteristics of the included studies; (2) Baseline characteristics of studied populations; and (3) Study outcomes. All reviewers' independently extracted data from the included articles and any discrepancies were solved by discussion.

\section{Dealing with missing data:}

Missing Standard Deviation (SD) of mean change from baseline was calculated from standard error or 95\% Confidence Interval (CI) according to Altman [22]

\section{Direct meta-analysis:}

Continuous outcomes were pooled as Mean Difference (MD) or Standardized Mean Difference (SMD) using inverse variance method, and dichotomous outcomes will be pooled as Relative Risk (RR) using Mantel-Haenszel method. The randomeffects method was used under the assumption of existing significant clinical and methodological heterogeneity. We performed all statistical analyses using Review Manager (RevMan) 5.3 or Open Meta-analyst for windows.

\section{Assessment of heterogeneity:}

We assessed heterogeneity by visual inspection of the forest plots, chi-square, and I-square tests. According to the recommendations of Cochrane Handbook of Systematic Reviews and metaanalysis, chi-square $p$-value less than 0.1 denote significant heterogeneity while I-square values show no important heterogeneity between $0 \%$ and $40 \%$, moderate heterogeneity from $30 \%$ to $60 \%$, substantial heterogeneity from $50 \%$ to $100 \%$. If any trials were judged to affect the homogeneity of the pooled estimates, we planned to perform a sensitivity analysis to assess outcomes with and without the trials that were affecting the homogeneity of the effect estimates.

\section{Results}

\section{I- Search results:}

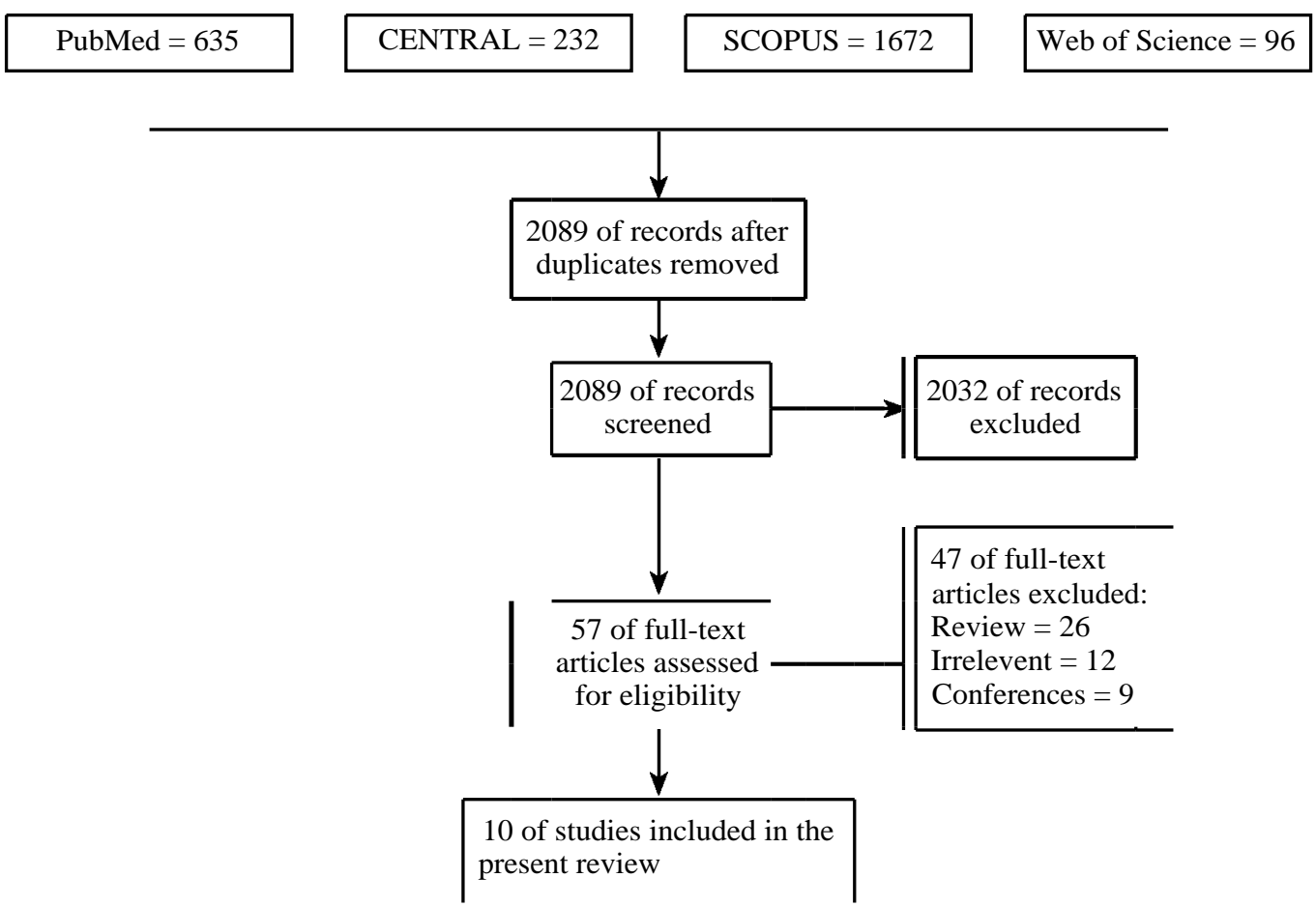

Fig. (1): PRISMA flow-chart. 


\section{II- Characteristics of the included studies (No. $=10$ studies):}

Table (1): Summary characteristics of the included studies.

\begin{tabular}{|c|c|c|c|c|c|c|c|c|c|}
\hline No. & Authors & Year & $\begin{array}{l}\text { Study } \\
\text { period }\end{array}$ & Country & Study design & Population & Intervention & No. & Main findings \\
\hline 1 & - Kim et al. & 2020 & $\begin{array}{c}\cdot 2001- \\
2013\end{array}$ & $\begin{array}{l}\text { - South } \\
\text { Korea }\end{array}$ & $\begin{array}{l}\text { - Retrospective } \\
\text { study }\end{array}$ & $\begin{array}{l}\text { - OBC patients } \\
\text { with negative } \\
\text { breast MRI }\end{array}$ & - $\mathrm{BCT}+\mathrm{ALND}$ & 66 & $\begin{array}{l}\text { - Patients with MRI-OBC were } \\
\text { successfully treated with } \\
\text { BCT }\end{array}$ \\
\hline 2 & - Ge et al. & 2018 & $\begin{array}{r}\cdot 2004- \\
2014\end{array}$ & - USA & $\begin{array}{l}\text { - Retrospective } \\
\text { study }\end{array}$ & - OBC patients & $\begin{array}{l}\text { - Mastectomy, BCS, } \\
\text { ALND, or SNLD }\end{array}$ & 479 & $\begin{array}{l}\text { - BCS plus ALND and } \\
\text { radiotherapy showed a } \\
\text { survival benefit that was } \\
\text { similar to that of mastectomy } \\
\text { for OBC patients }\end{array}$ \\
\hline 3 & $\begin{array}{l}\text { - Huang et } \\
\text { al. }\end{array}$ & 2017 & $\begin{array}{l}\text { - } 2005- \\
2016\end{array}$ & - China & $\begin{array}{l}\text { - Retrospective } \\
\text { study }\end{array}$ & $\begin{array}{l}\text { - Patients } \\
\text { diagnosed with } \\
\text { OBC, }\end{array}$ & $\begin{array}{l}\text { - Mastectomy with } \\
\text { ALND + RT; } \\
\text { Mastectomy with } \\
\text { ALND; } \\
\text { Quadrantectomy } \\
\text { with ALND + RT; } \\
\text { Quadrantectomy } \\
\text { with ALND. }\end{array}$ & 20 & $\begin{array}{l}\text { - Modified radical mastectomy } \\
+ \text { RT is still a safe and } \\
\text { effective choice }\end{array}$ \\
\hline 4 & $\begin{array}{l}\text { - Kemeny et } \\
\text { al. }\end{array}$ & 1986 & $\begin{array}{l}\text { - } 1973- \\
1985\end{array}$ & - USA & $\begin{array}{l}\text { - Retrospective } \\
\text { study }\end{array}$ & $\begin{array}{l}\text { - Patients } \\
\text { diagnosed with } \\
\text { OBC, }\end{array}$ & $\begin{array}{l}\text { - Mastectomy or } \\
\text { ALND }\end{array}$ & 24 & $\begin{array}{l}\text { - Mastectomy should not } \\
\text { automatically be performed }\end{array}$ \\
\hline 5 & $\begin{array}{l}\text { - Foroudi et } \\
\text { al. }\end{array}$ & 2000 & $\begin{array}{l}\text { - } 1979- \\
1996\end{array}$ & - Australia & $\begin{array}{l}\text { - Retrospective } \\
\text { study }\end{array}$ & $\begin{array}{l}\text { - Patients } \\
\text { diagnosed with } \\
\text { OBC, }\end{array}$ & $\begin{array}{l}\text { - Observation, } \\
\text { Mastectomy, or } \\
\text { Radiotherapy }\end{array}$ & 20 & $\begin{array}{l}\text { - Observation of the breast is } \\
\text { not a recommended option }\end{array}$ \\
\hline 6 & $\begin{array}{l}\text { - Vlastos et } \\
\text { al. }\end{array}$ & 2008 & $\begin{array}{l}\text { - } 1951- \\
1998\end{array}$ & - USA & $\begin{array}{l}\text { - Retrospective } \\
\text { study }\end{array}$ & - OBC patients & - Mastectomy or BCS & 45 & $\begin{array}{l}\text { - Occult primary carcinoma } \\
\text { with axillary metastases can } \\
\text { be treated with preservation } \\
\text { of the breast without a } \\
\text { negative impact on local } \\
\text { control or survival }\end{array}$ \\
\hline 7 & $\begin{array}{l}\text { - Varadarajan } \\
\text { et al. }\end{array}$ & 1997 & $\begin{array}{l}\cdot 1997- \\
2004\end{array}$ & - USA & $\begin{array}{l}\text { - Retrospective } \\
\text { study }\end{array}$ & - OBC patients & - Mastectomy or BCS & 12 & $\begin{array}{l}\text { - MR findings can influence } \\
\text { surgical treatment. }\end{array}$ \\
\hline 8 & - He et al. & 2012 & $\begin{array}{c}1998- \\
2010\end{array}$ & - China & $\begin{array}{l}\text { - Retrospective } \\
\text { study }\end{array}$ & - OBC patients & $\begin{array}{l}\text { - Mastectomy with } \\
\text { ALND + RT; } \\
\text { Mastectomy with } \\
\text { ALND; } \\
\text { Quadrantectomy } \\
\text { with ALND + RT; } \\
\text { Quadrantectomy } \\
\text { with ALND. }\end{array}$ & 95 & $\begin{array}{l}\text { - Patients with OBC who } \\
\text { received ALND and } \\
\text { subsequent breast } \\
\text { radiotherapy had similar } \\
\text { outcomes to patients who } \\
\text { underwent mastectomy. }\end{array}$ \\
\hline 9 & - Woo et al. & 2013 & $\begin{array}{c}1992- \\
2010\end{array}$ & $\begin{array}{l}\text { - South } \\
\text { Korea }\end{array}$ & $\begin{array}{l}\text { - Retrospective } \\
\text { study }\end{array}$ & - OBC patients & - Mastectomy or BCS & 40 & $\begin{array}{l}\text { - There was no difference } \\
\text { mastectomy, BCS, and No } \\
\text { Op } \pm \text { RT }\end{array}$ \\
\hline 10 & - Rueth et al. & 2015 & $\begin{array}{l}\cdot 2000- \\
2011\end{array}$ & - USA & $\begin{array}{l}\text { - Retrospective } \\
\text { study }\end{array}$ & - OBC patients & - Mastectomy or BCS & 36 & $\begin{array}{l}\text { - Breast conservation- } \\
\text { performed with } \\
\text { contemporary imaging and } \\
\text { multimodality treatment- } \\
\text { provides excellent local } \\
\text { control }\end{array}$ \\
\hline
\end{tabular}

Table (2): Baseline of the included studies.

\begin{tabular}{|c|c|c|c|c|c|c|c|c|c|c|}
\hline \multirow{2}{*}{ No. } & \multirow{2}{*}{ Authors } & \multirow{2}{*}{ Year } & \multirow{2}{*}{$\begin{array}{c}\text { Age }(\text { median }), \\
\text { yo }\end{array}$} & \multirow{2}{*}{$\mathrm{N}$} & \multirow{2}{*}{$\begin{array}{c}\text { Pre-operative } \\
\text { MRI }\end{array}$} & \multirow{2}{*}{$\begin{array}{l}\text { Primary tumor } \\
\text { found in } \\
\text { mastectomy } \\
\text { specimen }\end{array}$} & \multicolumn{3}{|c|}{ Adjuvant therapy } & \multirow{2}{*}{$\begin{array}{l}\text { Follow-up } \\
\text { (mo) }\end{array}$} \\
\hline & & & & & & & Chemotherapy & Endocrine & $\begin{array}{l}\text { Anti HER2 } \\
\text { therapy }\end{array}$ & \\
\hline 1 & Kim et al. & 2020 & $54(32-78)$ & 66 & $66(100 \%)$ & * & $63(96 \%)$ & $52(78.8 \%)$ & $12(18 . \%)$ & $82(10-178)$ \\
\hline 2 & Ge et al. & 2018 & $50(18-67)$ & 479 & $*$ & $*$ & $*$ & $*$ & $*$ & $*$ \\
\hline 3 & Huang et al. & 2017 & $44(38-60)$ & 20 & $*$ & $*$ & $12(60 \%)$ & $8(40 \%)$ & $*$ & $*$ \\
\hline 4 & Kemeny et al. & 1986 & $58(37-78)$ & 24 & $*$ & $5(45.4 \%)$ & $12(42.9 \%)$ & $*$ & $*$ & $45(9-144)$ \\
\hline 5 & Foroudi et al. & 2000 & $59.3(35-78)$ & 20 & $1(5 \%)$ & 0 & $9(45 \%)$ & $3(15 \%)$ & $*$ & $73(7-374)$ \\
\hline 6 & Vlastos et al. & 2008 & $54(32-79)$ & 45 & & $1(76.9 \%)$ & $31(68.9 \%)$ & $10(22.2 \%)$ & $*$ & $84(12-396)$ \\
\hline 7 & Varadarajan et al. & 1997 & $58(32-88)$ & 12 & $7(70 \%)$ & $*$ & $9(90 \%)$ & $6(60 \%)$ & $*$ & $57(16-84)$ \\
\hline 8 & He et al. & 2012 & $52(27-83)$ & 95 & $41(43.1 \%)$ & $20(31.3 \%)$ & $84(88.4 \%)$ & $37(38.9 \%)$ & $*$ & $38.2(4-160)$ \\
\hline 9 & Woo et al. & 2013 & $50(33-64)$ & 40 & $22(55 \%)$ & $*$ & $37(92.5 \%)$ & $17(42.5 \%)$ & $*$ & $71.5(5-205)$ \\
\hline 10 & Rueth et al. & 2015 & $55(39-73)$ & 36 & $33(91.7 \%)$ & $*$ & $34(94.4 \%)$ & $1(2.8 \%)$ & $*$ & $64(9-143)$ \\
\hline
\end{tabular}


Table (3): Management lines of the included studies.

\begin{tabular}{|c|c|c|c|c|c|c|c|c|}
\hline \multirow{2}{*}{ No. } & \multirow{2}{*}{ Authors } & \multirow{2}{*}{ Year } & \multicolumn{6}{|c|}{ No. } \\
\hline & & & ALND + XRT & Mastectomy & ALND + mastectomy & ALND & $\mathrm{ALND}+\mathrm{BCS}$ & XRT \\
\hline 1 & Kim et al. & 2020 & None & None & None & None & 66 & 63 \\
\hline 2 & Ge et al. & 2018 & $*$ & $*$ & $*$ & $*$ & $*$ & $*$ \\
\hline 3 & Huang et al. & 2017 & None & 10 & None & None & 10 & None \\
\hline 4 & Kemeny et al. & 1986 & 2 & None & 11 & 5 & None & None \\
\hline 5 & Foroudi et al. & 2000 & 11 & None & 2 & 5 & None & 2 \\
\hline 6 & Vlastos et al. & 2008 & 25 & None & 13 & 7 & None & None \\
\hline 7 & Varadarajan et al. & 1997 & 8 & None & 1 & None & 1 & None \\
\hline 8 & He et al. & 2012 & 13 & None & 64 & 18 & None & None \\
\hline 9 & Woo et al. & 2013 & 11 & None & 12 & None & 17 & None \\
\hline 10 & Rueth et al. & 2015 & 24 & None & 9 & None & None & 3 \\
\hline
\end{tabular}

Table (4): Management lines of the included studies.

Locoregional recurrence

Distant metastasis

Mortality

\begin{tabular}{|c|c|c|c|c|c|c|c|c|c|c|c|c|}
\hline Authors & Year & $\begin{array}{l}\text { ALND } \\
+ \text { XRT }\end{array}$ & $\begin{array}{c}\text { ALND + } \\
\text { mastectomy }\end{array}$ & ALND & $\begin{array}{l}\text { ALND } \\
+ \text { BCS }\end{array}$ & $\begin{array}{l}\text { ALND } \\
+ \text { XRT }\end{array}$ & $\begin{array}{c}\text { ALND + } \\
\text { mastectomy }\end{array}$ & ALND & $\begin{array}{l}\text { ALND } \\
+ \text { XRT }\end{array}$ & $\begin{array}{c}\text { ALND + } \\
\text { mastectomy }\end{array}$ & $\begin{array}{l}\text { ALND } \\
+ \text { BCS }\end{array}$ & ALND \\
\hline Kim et al. & 2020 & None & None & None & $9(13.6 \%)$ & None & None & $5(7.9 \%)$ & None & None & None & $(6.6 \%)$ \\
\hline $\begin{array}{l}\text { Ge et al. } \\
\text { Huang et al. }\end{array}$ & 2017 & None & $1(10 \%)$ & None & $3(30 \%)$ & None & None & None & & $5(50 \%)$ & $5(50 \%)$ & None \\
\hline Kemeny et al. & 1986 & 0 & 0 & 0 & & 0 & 0 & $1(20 \%)$ & $1(50 \%)$ & $4(36.7 \%)$ & & 0 \\
\hline Foroudi et al. & 2000 & $6(54.5 \%)$ & 0 & $5(100 \%)$ & & * & * & * & $1(9.1 \%)$ & 0 & & $2(40 \%)$ \\
\hline Vlastos et al. & 2008 & $2(8 \%)$ & $2(15.4 \%)$ & $2(28.6 \%)$ & & $3(12 \%)$ & $4(30.7 \%)$ & $4(57.1 \%)$ & $3(12 \%)$ & $3(23.1 \%)$ & & $4(57.1 \%)$ \\
\hline Varadarajan et al. & 1997 & 0 & 0 & None & & 0 & 0 & None & 0 & 0 & & None \\
\hline He et al. & 2012 & $1(7.7 \%)$ & $7(10.9 \%)$ & $5(27.8 \%)$ & & $2(15.4 \%)$ & $6(9.4 \%)$ & $1(5.7 \%)$ & $3(23.1 \%)$ & $10(15.6 \%)$ & & $5(27.8 \%)$ \\
\hline Woo et al. & 2013 & $3(27.8 \%)$ & $2(16.7 \%)$ & None & & 0 & $4(33.3 \%)$ & None & $1(9.1 \%)$ & $3(25 \%)$ & & None \\
\hline Rueth et al. & 2015 & 0 & 0 & None & & $1(4.2 \%)$ & 0 & None & 0 & 0 & & None \\
\hline
\end{tabular}

Table (4): Management lines of the included studies (Cont.).

\begin{tabular}{|c|c|c|c|c|c|c|c|c|c|c|c|c|}
\hline \multirow[b]{2}{*}{ Authors } & \multirow[b]{2}{*}{ Year } & \multicolumn{4}{|c|}{ OS } & \multicolumn{4}{|c|}{ DFS } & \multicolumn{3}{|c|}{ BCSS } \\
\hline & & $\begin{array}{l}\text { ALND } \\
+ \text { XRT }\end{array}$ & $\begin{array}{l}\text { ALND + } \\
\text { mastec- } \\
\text { tomy }\end{array}$ & ALND & $\begin{array}{l}\text { ALND } \\
+ \text { BCS }\end{array}$ & $\begin{array}{l}\text { ALND } \\
+ \text { XRT }\end{array}$ & $\begin{array}{l}\text { ALND + } \\
\text { mastec- } \\
\text { tomy }\end{array}$ & ALND & $\begin{array}{l}\text { ALND } \\
+ \text { BCS }\end{array}$ & $\begin{array}{l}\text { ALND } \\
+ \text { XRT }\end{array}$ & $\begin{array}{l}\text { ALND + } \\
\text { mastec- ALND } \\
\text { tomy }\end{array}$ & $\begin{array}{l}\text { ALND } \\
+ \text { BCS }\end{array}$ \\
\hline - Kim et al. & 2020 & None & None & None & $62(93.2 \%)$ & None & None & None & $61(92.1 \%)$ & $61(92.1 \%)$ & & $63(96 \%)$ \\
\hline - Ge et al. & 2018 & & \multicolumn{2}{|c|}{ None } & & \multicolumn{4}{|c|}{ None } & \multicolumn{3}{|c|}{$85.50 \%$} \\
\hline - Huang et al. & 2017 & None & $5(50 \%)$ & None & $5(50 \%)$ & \multicolumn{4}{|c|}{ None } & \multicolumn{3}{|c|}{ None } \\
\hline - Kemeny et al. & 1986 & $1(50 \%)$ & $7(63.3 \%)$ & $5(100 \%)$ & None & \multicolumn{4}{|c|}{ None } & \multicolumn{3}{|c|}{ None } \\
\hline - Foroudi et al. & 2000 & $11(90.9 \%)$ & $2(100 \%)$ & None & None & \multicolumn{4}{|c|}{ None } & \multicolumn{3}{|c|}{ None } \\
\hline - Vlastos et al. & 2008 & $29(88 \%)$ & $9(76.9 \%)$ & None & None & \multicolumn{4}{|c|}{ None } & \multicolumn{3}{|c|}{ None } \\
\hline $\begin{array}{l}\text { - Varadarajan } \\
\text { et al. }\end{array}$ & 1997 & None & None & None & None & \multicolumn{4}{|c|}{ None } & \multicolumn{3}{|c|}{ None } \\
\hline - He et al. & 2012 & None & None & None & None & \multicolumn{4}{|c|}{ None } & \multicolumn{3}{|c|}{ None } \\
\hline • Woo et al. & 2013 & $87.50 \%$ & None & $74.00 \%$ & $72.00 \%$ & \multicolumn{4}{|c|}{$74.90 \%$} & \multicolumn{3}{|c|}{$76.90 \%$} \\
\hline - Rueth et al. & 2015 & & & & & \multicolumn{4}{|c|}{ None } & \multicolumn{3}{|c|}{ None } \\
\hline
\end{tabular}

\section{III- Meta-analysis results:}

\section{1- Locoregional recurrence:}
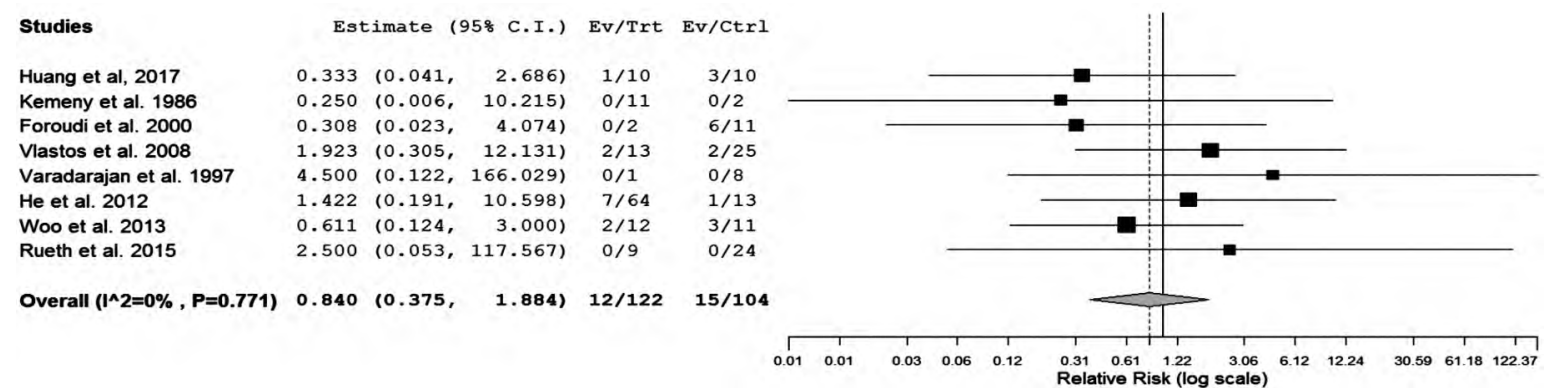

Fig. (2): Forest plot of rates of locoregional recurrence (mastectomy versus BCS). 
Overall, eight studies reported the rates of locoregional recurrence. The overall effect estimates showed no significant difference between mastectomy and BCS regarding locoregional re- currence $(\mathrm{RR}=0.84,95 \%$ CI $0.375-1.88)$. The pooled studies showed no significant heterogeneity $\left[p=0.77 ; \mathrm{I}^{2}=0 \%\right.$; Fig. (2)].

2- Distant metastasis:
Studies

Kemeny et al. 1986 Vlastos et al. 2008 Varadarajan et al. 1997 He et al. 2012 Woo et al. 2013 Rueth et al. 2015

Overall (I^2=0\%,P=0.436) $1.501 \quad(0.643, \quad 3.504) \quad 14 / 110$

\begin{tabular}{|c|c|c|c|c|}
\hline .250 & $(0.006$, & $10.215)$ & $0 / 11$ & $0 / 2$ \\
\hline 2.564 & $(0.672$, & $9.778)$ & $4 / 13$ & $3 / 25$ \\
\hline 4.500 & $(0.122$, & $166.029)$ & $0 / 1$ & $0 / 8$ \\
\hline 0.609 & (0.138, & $2.691)$ & $6 / 64$ & $2 / 13$ \\
\hline 8.308 & (0.498, & $138.601)$ & $4 / 12$ & $0 / 11$ \\
\hline 0.833 & $(0.037$, & $18.786)$ & $0 / 9$ & $1 / 24$ \\
\hline & 10.043 & $3.504)$ & $14 / 110$ & $6 / 8$ \\
\hline
\end{tabular}

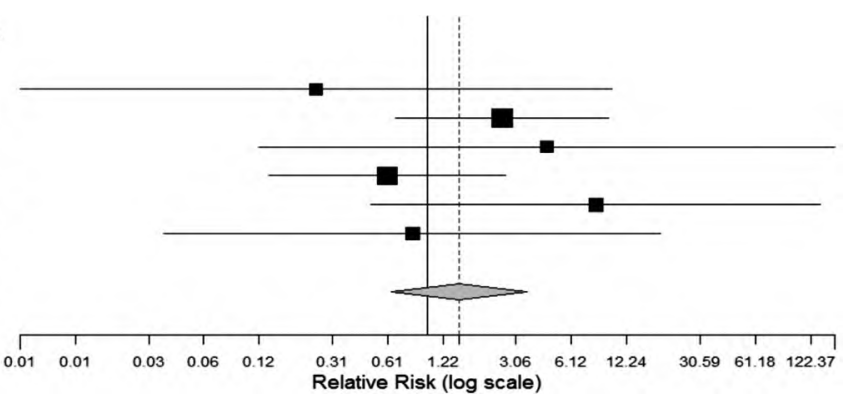

Fig. (3): Forest plot of rates of distant metastasis (mastectomy versus BCS).

Seven studies reported the rates of distant metastasis. The overall effect estimates showed no significant difference between mastectomy and $\mathrm{BCS}$ regarding distant metastasis $(\mathrm{RR}=1.5,95 \%$
CI 0.64-3.1). The pooled studies showed no significant heterogeneity $\left[p=0.43 ; \mathrm{I}^{2}=0 \%\right.$; Fig. (3)].

\section{3- Mortality:}

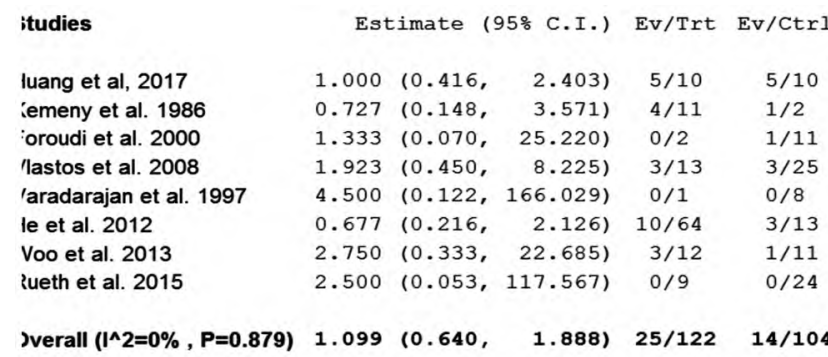

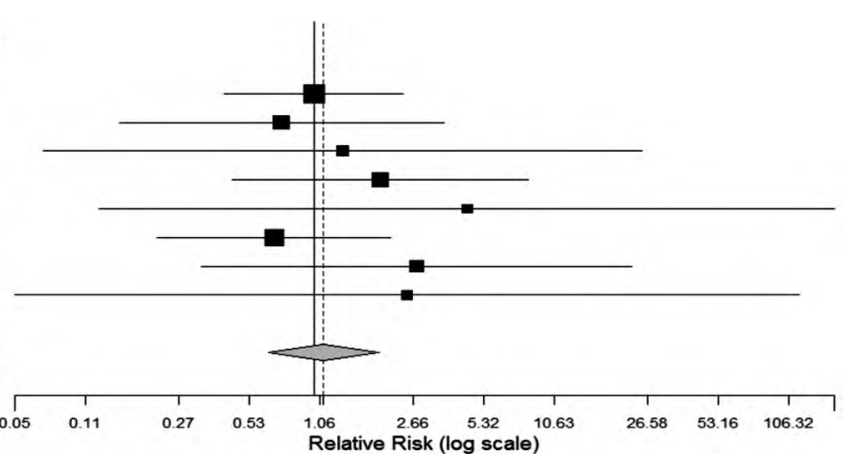

Fig. (4): Forest plot of rates of mortality (mastectomy versus BCS).

Overall, eight studies reported the rates of mortality. The overall effect estimates showed no significant difference between mastectomy and BCS regarding mortality $(\mathrm{RR}=1.1,95 \%$ CI 0.64 1.88). The pooled studies showed no significant heterogeneity $\left[p=0.97 ; \mathrm{I}^{2}=0 \%\right.$; Fig. (4)].

\section{Discussion}

Breast cancer is the commonest primary neoplasm affecting females in Egypt, it is considered the third leading cause of cancer related deaths in Egypt, it has been established during past decades, that surgery, radiotherapy and chemotherapy are corner stones in treatment of breast cancer [23] .

Occult Breast Cancer (OBC) is a rare entity of breast cancer which represents less than $1 \%$ of the cases [24]. In such cases, metastatic axillary carcinoma arise in the nonexistence of clinically identifiable breast tumor (TON+ve) in addition no radiologically, or pathologically evidence of breast mass [25]. OBC presents both diagnostic and ther- apeutic difficulty due to the scarcity of cases and relatively limited surgical experience. Few retrospective studies have been available to point out practice patterns, and it is believed that prospective series will be difficult to perform as low number of cases can be colledtec over a great period of time [26]

It is believed that OBC has an unknown oncologic behavior. Studies suggests that the survival outcomes of OBC are similar or slightly better compared with breast cancer of matching nodal status. However, only few studies have demonstrated worse outcomes of OBC [27]

Management of OBC originally favored axillary lymph node dissection plus mastectomy. However, there have been a series reporting the use of breast conserving surgery plus ALND followed by whole breast radiotherapy and observation of the breast. Findings from the analysis of SEER database demonstrated higher rates of stage III disease, N3 disease, hormone receptors negativity and Her2 
receptor positivity [28]. Halsted supported mastectomy and ALND in the initial report of OBC [11] This remained the backbone of $\mathrm{OBC}$ treatment for many years, however there are some reports of favoring BCS, as upper outer quadrantectomy as this was expected to represent the most probable location to find an OBC. The Korean Breast Cancer Society showed that ALND plus BCS followed by WBRT had comparable survival to Mastectomy plus ALND [24]. A third approach was to utilize WBRT in addition to ALND. A survey for American breast surgeons opinion regarding OBC management conducted in 2005 demonstrated that in order to treat OBC $43 \%$ opted for mastectomy, $37 \%$ whole breast radiation and $6 \%$ would not treat the breast [29].

In this meta-analysis, we systematically investigated the present therapeutic options in patients diagnosed with $\mathrm{OBC}$, targeting a definition of the surgical approach associated with best surgical and recurrence outcomes.

10 studies were eligible for inclusion in our meta-analysis, all were published in the period from inception till 2020, revising all these data, nonetheless designing meta-analysis study to evaluate the overall result and to highpoint the different surgical approaches in management of occult breast cancer and to evaluate recurrence rate and prognosis.

A total of 837 patients were included in metaanalysis, with median age 54 years range from 44 to 59.3 years old with median follow-up period 67.7 months, compared to other meta-analysis conducted by Macedo et al., had seven studies included with total number of 241 patients eligible for analysis, median age were 55 years ranged between 50-59 years old, nonetheless the median follow-up period was 61.7 months ranged from 38 to 84 months [30].

Only 8 studies included survival data of patients included, comparing between OS and DFS of patients who underwent mastectomy plus ALND representing $42.4 \%(n=112)$, patients who underwent BCS plus ALND followed by radiotherapy $35.6 \%(n=94)$ and who received only axillary radiation therapy $25.7 \%(n=68)$.

Regarding loco regional recurrence, currant study didn't show any superiority of neither BCS nor mastectomy in terms of loco regional recurrence with $p$-value 0.77 and RR 0.84 .

Adding to that only seven studies reported distant metastasis as a primary end point, however there was no significant distant recurrence difference between 2 groups with $p$-value 0.43 and RR 1.5 , these results was confirmed by Macedo et al., as in his meta analysis there was no significant difference in loco regional or distant recurrence with $p$-value 0.78 and 0.16 respectively [30].

The overall mortality rates of our study was $15.8 \%(n=45)$ during the period of follow-up, for those who underwent BCS plus ALND were $1.7 \%$ $(n=5)$, nonetheless for patients who underwent mastectomy plus ALND, it was $8.8 \%(n=25)$ with $p$-value 0.97 and RR 1.1. Comparing these results to study conducted by Macedo et al., there was no statistically significant difference in mortality rates between two surgical approaches with $p$-value 0.65 and RR 0.85 [30].

\section{Conclusion:}

- There was no statistically difference between BCS + ALND and Mastectomy + ALND surgical approaches in terms of recurrence including loco regional and distant.

- There was difference in survival (OS and DFS) between BCS + ALND and Mastectomy + ALND surgical approaches.

\section{References}

1- SMITH R.A., ANDREWS K., BROOKS D., DeSANTIS C.E., FEDEWA S.A., LORTET-TIEULENT J. and WENDER R.C.: Cancer screening in the United States, 2016: A review of current American Cancer Society guidelines and current issues in cancer screening. CA: A cancer journal for clinicians, 66 (2): 95-114, 2016.

2- MERKEL A.R., RUPPENDER N. S., MARTIN T.J., MUNDY G.R., STERLING J.A. and GUELCHER S.A.: Matrix rigidity induces osteolytic gene expression of metastatic breast cancer cells. PloS one, 5 (11): e15451, 2010.

3- SIU A.L.: Screening for breast cancer: US Preventive Services Task Force recommendation statement. Annals of internal medicine, 164 (4): 279-96, 2016.

4- CADOO K.A., FORNIER M.N. and MORRIS P.G.: Biological subtypes of breast cancer: Current concepts and implications for recurrence patterns. The quarterly journal of nuclear medicine and molecular imaging: Official publication of the Italian Association of Nuclear Medicine (AIMN) [and] the International Association of Radiopharmacology (IAR), [and] Section of the Society of..., 57 (4): 312-21, 2013.

5- SOLOMAYER E.F., DIEL I.J., MEYBERG G.C., GOLLAN C.H. and BASTERT G.: Metastatic breast cancer: Clinical course, prognosis and therapy related to the first site of metastasis. Breast cancer research and treatment, 59 (3): 271-8, 2000.

6- SORLIE T., PEROU C.M., TIBSHIRANI R., AAS T. GEISLER S., JOHNSEN H. and THORSEN T.: Gene expression patterns of breast carcinomas distinguish tumor subclasses with clinical implications. Proceedings of the National Academy of Sciences, 98 (19): 10869-74, 2011. 
7- KENNECKET H., YERUSHALMI R., WOODS R., et al.: Metastatic behavior of breast cancer subtypes. J. Clin. Oncol., 28: 3271-327, 2010.

8- SMID M., WANG Y., ZHANG Y., SIEUWERTS A.M., YU J., KLIJN J.G. and MARTENS J.W.: Subtypes of breast cancer show preferential site of relapse. Cancer Research, 68 (9): 3108-14, 2008.

9- Collaborative Group on Hormonal Factors in Breast Cancer: Familial breast cancer: collaborative reanalysis of individual data from 52 epidemiological studies including 58209 women with breast cancer and 101986 women without the disease. The Lancet, 358 (9291): 1389-99, 2001.

10- PAGE K., HAVA N., WARD B., BROWN J., GUTTERY D.S., RUANGPRATHEEP C. and SHAW J.A.: Detection of HER2 amplification in circulating free DNA in patients with breast cancer. British Journal of Cancer, 104 (8): 1342-8, 2011.

11- HALSTED W.S.I.: The results of radical operations for the cure of carcinoma of the breast. Ann. Surg., 46: 1-19, 1907.

12-KINOSHITA S., HIRANO A., KOBAYASHI S., KOMINE K., KYODA S., et al.: Metachronous secondary primary occult breast cancer initially presenting with metastases to the contralateral axillary lymph nodes: Report of a case. Breast Cancer, 17: 71-4, 2010.

13- WALKER G.V., SMITH G.L., PERKINS G.H., OH J.L., WOODWARD W., YU T.K., HUNT K.K., HOFFMAN K., STROM E.A. and BUCHHOLZ T.A.: Population based analysis of occult primary breast cancer with axillary lymph node metastasis. Cancer, 116 (17): 4000-6, 2010.

14-De BRESSE J., De VOS B., VANDER E.F. and HULSEWE K.: Breast MRI in clinically and mammographically occult breast cancer presenting with an axillary metastasis: A systematic review. Eur. J. Surg. Oncol., 36: 114-9, 2010.

15- ZHU Y., LUO M., JIA Z. and GUO J.F.: Diagnoses and therapy of occult breast cancer: A systematic review. J. Mol. Biomark. Diagn., 2: 023, 2016.

16- WORMALD R., SHEAHAN P. and TIMON C.: A case of head and neck cancer metastasizing to the axillary lymph nodes. Ear. Nose Throat. J., 89: E24-26, 2010.

17- KIMBROUGH C.W., McMASTERS K.M., QUILLO A. and AJKAY N.: Occult metastases in node-negative breast cancer: A Surveillance, Epidemiology, and End Resultsbased analysis. Surgery, 158: 494-500, 2015.

18- CHOUDHURY M., AGRAWAL S., PUJANI M. and THOMAS S.: Immunohistochemical detection of axillary lymph node micrometastases in node negative breast cancer patients using cytokeratin and epithelial membrane antigen. South Asian J. Cancer, 4: 28-31, 2015.
19- OLSON J.A., MORRIS E.A., VAN ZEE K.J., LINEHAN D.C. and BORGEN P.I.: Magnetic resonance imaging facilitates breast conservation for occult breast cancer. Ann. Surg. Oncol., 7: 411-5, 2000.

20- KUHL C.K., MORAKKABATI N., LEUTNER C.C., SCHMIEDEL A., WARDELMANN E., et al.: MR imaging: Guided large-core (14-gauge) needle biopsy of small lesions visible at breast MR imaging alone. Radiology, 220: 31-9, 2001.

21- MOHER D., LIBERATI A. and TJ A.D.: The PRISMA Group. Preferred Reporting Items for Systematic Reviews and Meta-Analyses: The PRISMA Statement. Ann. Intern. Med., 151: 264-9, 2009.

22- ALTMAN D.G.: Why we need confidence intervals. World journal of surgery, 29 (5): 554-6, 2005.

23- BRAY L.J., SECKER C., MUREKATETE B., SIEVERS J., BINNER M., WELZEL P.B. and WERNER C.: Threedimensional in vitro hydro-and cryogel-based cell-culture models for the study of breast-cancer metastasis to bone. Cancers, 10 (9): 292, 2018.

24- SOHN G., SON B.H., LEE S.J., et al.: Treatment and survival of patients with occult breast cancer with axillary lymph node metastasis: A nationwide retrospective study. J. Surg. Oncol., 110: 270-4, 2014.

25- MONTAGNA E., BAGNARDI V., ROTMENEZ N., et al.: Immunohisto-chemically defined subtypes and outcome in occult breast carcinoma with axillary presentation. Breast Cancer Res. Treat., 129: 867-75, 2011.

26- WILD J.B., THRUSH S. and VIDYA R.: 'What can National Registry data tell us about occult breast cancer?', Chinese Clinical Oncology, 8 (8): 2-3, 2019.

27- HUANG K.Y., ZHANG J., FU W.F., LIN Y.X. and SONG C.G.: Different Clinicopathological Characteristics and Prognostic Factors for Occult and Non-occult Breast Cancer: Analysis of the SEER Database. Frontiers in Oncology, 10: 1420, 2020.

28- GE L.P., LIU X.Y., XIAO Y., GOU Z.C., ZHAO S., JIANG Y.Z. and DI G.H.: Clinicopathological characteristics and treatment outcomes of occult breast cancer: A SEER population-based study. Cancer Management and Research; 10: 4381, 2018.

29- KHANDELWAL A.K. and GARGUILO G.A.: Therapeutic options for occult breast cancer: A survey of the American Society of Breast Surgeons and review of the literature. The American Journal of Surgery, 190 (4): 609-13, 2005.

30- MACEDO S., NG C.K., MURRAY M.P., GUERINIROCCO E., MARTELOTTO L.G., GEYER F.C., BIDARD F.C., BERMAN S., FUSCO N., SAKR R.A. and EBERLE C.A.: The genomic landscape of male breast cancers. Clinical Cancer Research, 22 (16): 4045-56, 2016. 


\section{دراسة مرتجعة للمقارنة ما بين نتائج طرق العلاج المختلفة

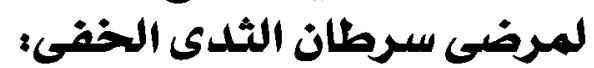

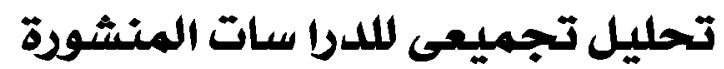

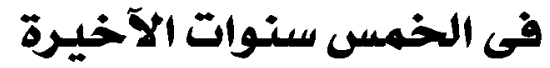

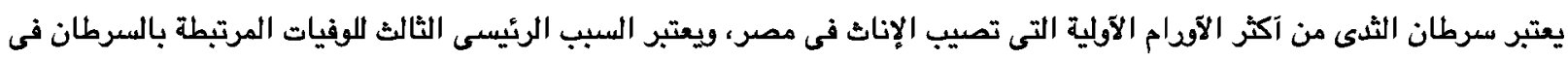

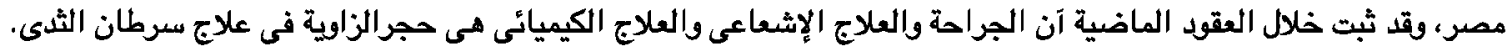

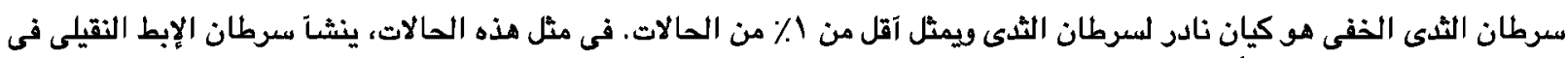

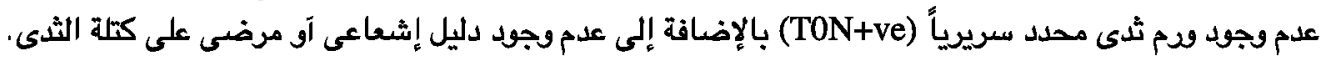

في هذا التحليل الثلوى، بحثا بشكل منهجى في الخيارات العلاجية الحالية للمرضى الذيم تم تشخيصهم بسرطان الثىى الذفى، مستهدفين

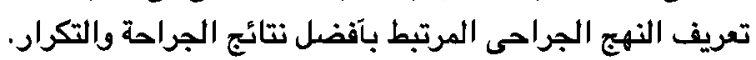

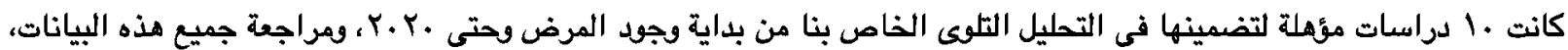

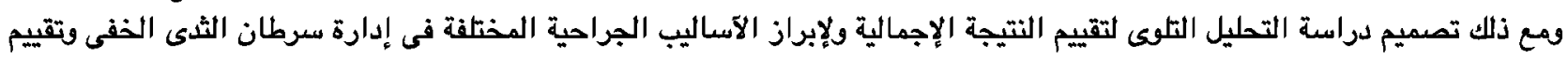

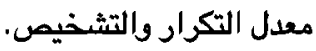

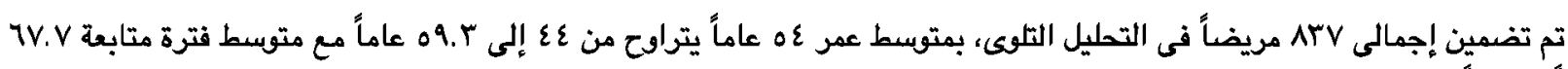

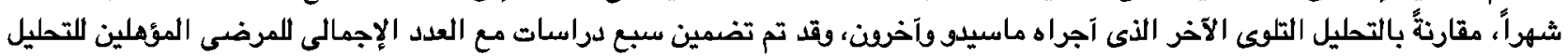

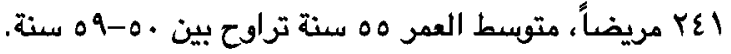

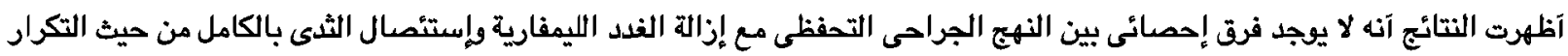

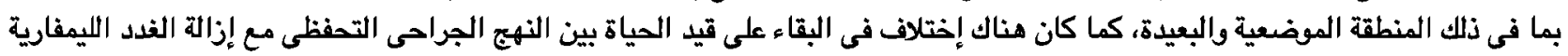
وإستئصال الثدى. 